\title{
Downward Terrestrial Gamma-ray Flashes at the Pierre Auger Observatory?
}

\author{
Roberta Colalillo ${ }^{a, b, *}$ on behalf of the Pierre Auger ${ }^{b}$ Collaboration \\ (a complete list of authors can be found at the end of the proceedings) \\ a INFN, Sezione di Napoli, Via Cintia, 80126 Napoli, Italy \\ ${ }^{b}$ Università degli Studi di Napoli "Federico II", Dipartimento di Fisica "E. Pancini”, Via Cintia, \\ 80126 Napoli, Italy \\ ${ }^{c}$ Observatorio Pierre Auger, Av. San Martín Norte 304, 5613 Malargüe, Argentina \\ E-mail: spokespersons@auger.org
}

At the Pierre Auger Observatory, designed primarily to study ultra-high-energy cosmic rays, phenomena related to atmospheric electricity are also observed. Particularly, events have been detected with the surface detector, characterized by long-lasting signals (tens of microseconds) and event footprints much larger (up to $200 \mathrm{~km}^{2}$ ) than those produced by the highest energy cosmic rays. Moreover, some of them appear to be accompanied by smaller events occurring in the same area within about $1 \mathrm{~ms}$ and probably produced by the same phenomenon. A previously reported correlation with the World Wide Lightning Location Network, as well as the observation of very low-altitude clouds, confirm that such events are related to thunderstorms. An ad-hoc reconstruction points to high-energy particles being produced very close to the ground, suggesting that they originate from electrons accelerated to relativistic energies in strong electric fields inside low clouds, as is the case for terrestrial gamma-ray flashes above thunderstorms.

A clear explanation of the observed phenomenon is hindered by two facts. One is that the rate of such events, detected serendipitously, is very small (less than 2 events/year) and decreases further after optimization of the surface detector trigger for low-energy shower-events. The second is that most events show a puzzling lack of signals in the central part of the footprint. We have studied in detail both effects and will present such studies here. We developed a strategy for a dedicated trigger to enhance the detection efficiency for these events associated with atmospheric-electricity events.

$37^{\text {th }}$ International Cosmic Ray Conference (ICRC 2021)

July 12th - 23rd, 2021

Online - Berlin, Germany

\footnotetext{
*Presenter
} 


\section{Atmospheric electricity phenomena at the Pierre Auger Observatory: $S D$-rings}

The Pierre Auger Observatory [1], designed to detect extensive air showers produced by ultrahigh energy cosmic rays in the atmosphere, can also be used to observe transient phenomena related to atmospheric electricity. In the fluorescence detector, used to record the evolution of showers in the atmosphere, peculiar events, associated with elves [2,3], a type of transient luminous events occurring in the upper atmosphere above thunderstorms, were discovered serendipitously. In the surface detector (SD), formed by 1600 water-Cherenkov detectors used to sample shower electrons, photons and muons, peculiar events have also been observed serendipitously during thunderstorms. Their features, in footprints and signals, are very different from those produced even by the highestenergy cosmic rays $[4,5]$.

At the Telescope Array (TA), the other large cosmic-ray observatory located in the northern hemisphere, large signals have also been detected with its scintillators at the time of thunderstorms [6]. There are differences and similarities between our events and TA events, whose origin is related to downward Terrestrial Gamma-ray Flashes (TGFs).

A TGF is a burst of high-energy photons originating in thunderstorms [7]. The emission is generated, via bremsstrahlung, by energetic runaway electrons accelerated by the electric fields in thunderclouds. TGFs have been routinely observed from space, but, in the last years, evidence of downward TGFs, occurring during strong initial breakdown pulses in the first few milliseconds of negative cloud-to-ground and low-altitude intra-cloud flashes, has been reported $[6,8]$. The propagation mechanisms of lightning are poorly understood. Lightning-leaders do not propagate in a continuous manner, but instead progress in a series of discrete "steps". The typical duration of the process is of the order of a millisecond, with the inter-step intervals last some tens of $\mu$ s. TGFs can easily saturate detectors far from the source.

At the Pierre Auger Observatory, the first peculiar event was detected in 2005. In the complete data sample, recorded since 2004, 23 events with the same characteristics have been identified. In the left panel of Fig. 1, the footprint of one of such events (No. 4067414) is compared with that of a very energetic cosmic ray $\left(E \approx 1.4 \times 10^{20} \mathrm{eV}\right)$ which triggered 17 stations. In the peculiar event, the footprint is much larger, with 74 triggered stations. The signals are also very different from those from air showers, with much larger duration. An example of a signal, lasting tens of $\mu$ s, is shown in the panel (f) of Fig. 2: it is an order of magnitude longer than the ones seen in cosmic-ray events (panels (a) and (b)). Moreover, in 2005, it was noted that also stations with high-frequency noise, ("lightning stations", panel (d) of Fig. 2), were part of such event, suggesting that lightning activity had happened at the same time. This hypothesis was verified by finding a correlation between the peculiar events and lightning strikes collected by the World Wide Lightning Location Network (WWLLN). As the Observatory makes use of the atmosphere as a giant calorimeter, several atmospheric-monitoring facilities are available, such as lasers, LIDARs, cloud cameras [11]. Although no atmospheric data were available at the exact time of the event shown in Fig. 1, the cloud cameras, operating only during the nightly data-taking of the FD, reported a $100 \%$ cloud coverage some hours before and/or after the event, with clouds estimated from lasers and LIDARs to be $\sim 2 \mathrm{~km}$ above ground level, suggesting that it occurred during bad weather and with very low clouds.

The most puzzling feature of this event, and of other peculiar events, is the lack of signals in 

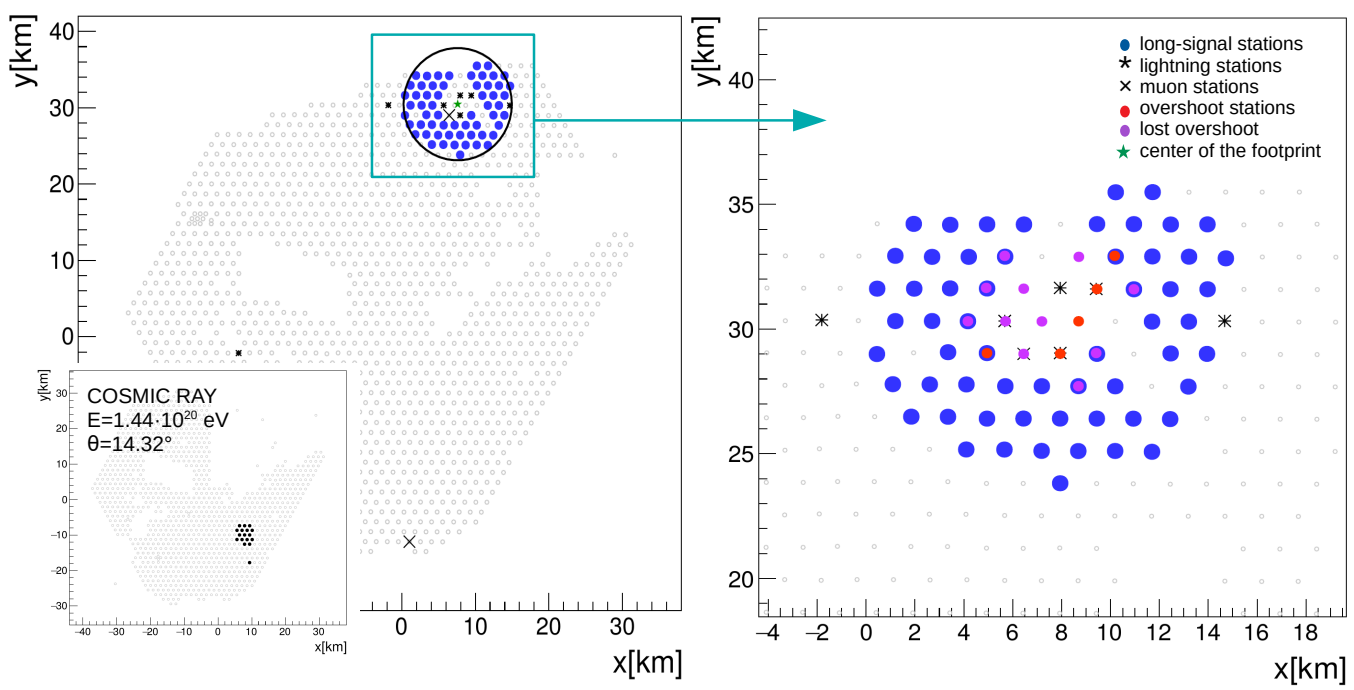

Figure 1: Left: Typical footprint of an SD-ring compared to that of a very high-energy cosmic ray. Right: Zoom of the SD-ring footprint: long-signal stations are marked in blue, lightning stations with stars, muon stations with crosses. Red and magenta dots represent, respectively, the overshoot stations and the stations in error state at the overshoot time ("lost overshoot stations") in an event registered about $500 \mu$ s after the SD-ring.

the center of the footprint. We refer to these as $S D$-rings. Investigations, described below, have established that this feature is an artifact arising from the recording system that was, of course, optimized for the detection of air showers. Accordingly a new trigger to change the read-out of the events has been devised (Sec. 4) and a new approach is being developed to understand the origin of these peculiar events.

\section{SD rings revisited}

Premise The revisiting of the $S D$-rings was guided by the hypothesis that the lack of signals in the center is due either to electronics, or trigger or data acquisition, or post-acquisition processing, or any combination of these. All systems are optimised for the rate, shape and signals of showers generated by ultra-high energy cosmic rays. Details are in [1] and in [9] and only a summary of relevant elements is given here.

The Cherenkov signals in each SD station are collected by three photomultipliers and digitised using $40 \mathrm{MHz}, 10$-bit Flash Analog-to-Digital Converters (FADCs). Based on these signals, the local data acquisition generates low-level triggers, at $\sim 20 \mathrm{~Hz}$. Such local triggers have been designed to respond to signals typical of air showers, i.e., to large signals not necessarily spread in time, such as those close to the core, or to sequences of small signals spread in time, such as those near the core in low-energy showers, or far from the core in high-energy ones. When the station signal satisfies one of the local trigger conditions, a time block of $19.2 \mu$ s from the FADC is copied to a buffer, in "stand-by" for a possible shower trigger. The block length has been adapted to the signal duration of a shower, taking, as a conservative reference, the length of a signal expected in a shower of $10^{21} \mathrm{eV}$ far from the core. The timing of each local trigger is sent to the central data acquisition system (CDAS), which searches for spatial and temporal correlations among all triggered stations. Once a 

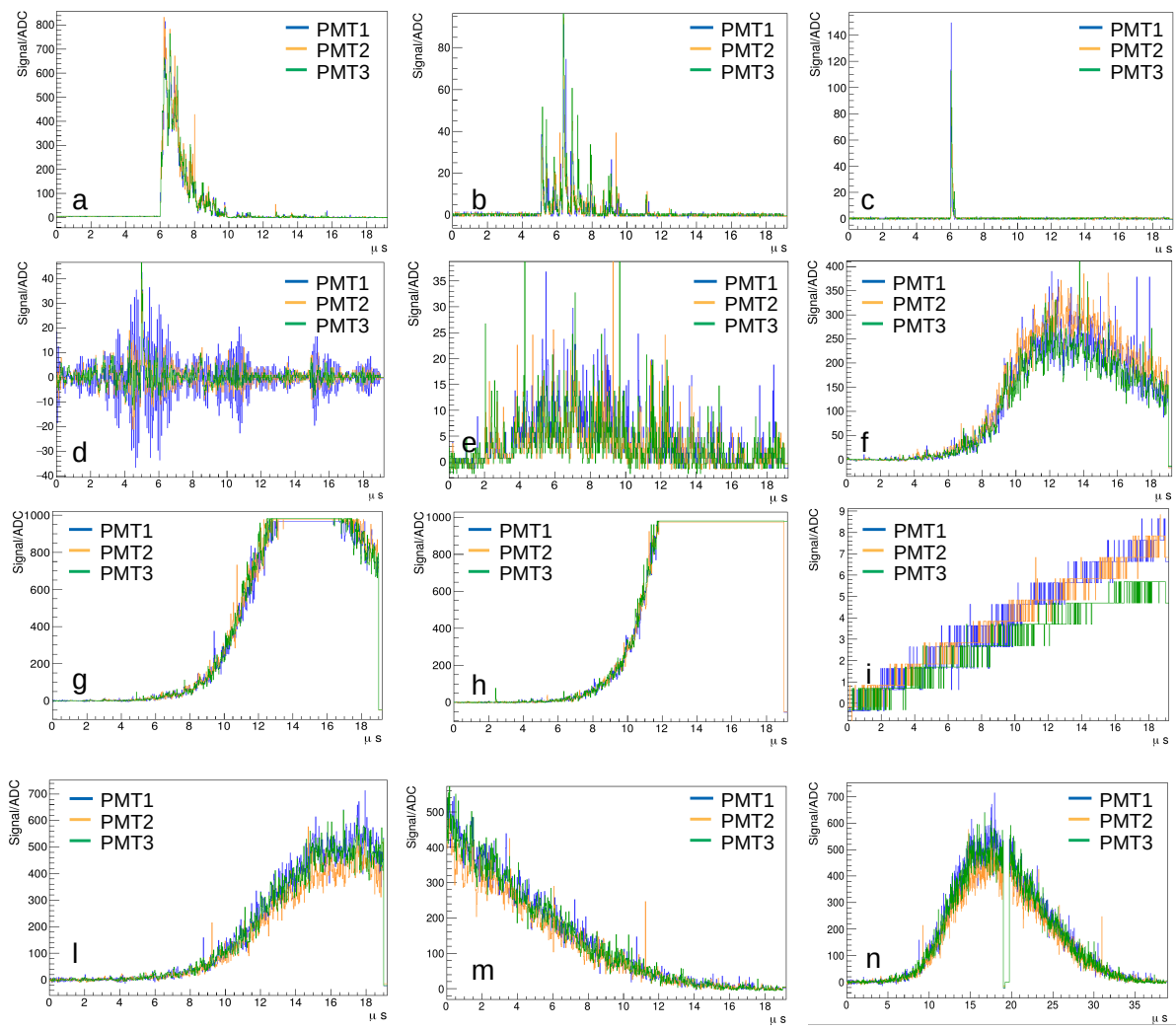

Figure 2: Collection of signals observed in events detected with the SD stations. The three different colors represent the signals from the 3 photomultipliers looking down in the water in each station. (a) and (b) are signals typical of showers, close to (a) and far from (b) the core, respectively. (c) is a signal due to a cosmic muon, usually found in accidentally triggered stations. (d) is a so-called lightning signal, dominated by high frequency noise. (e, f, g, h, l, m, and n), are examples of the so-called long signals: (e) and (f) are complete signals, (g) and (h) are saturated signals, (l) and (m) are the rising and falling portion of a very long signal which is not contained in a unique trace, ( $n$ ) is the result of the combination of (l) and (m). Finally, (i) is a so-called overshoot, an electronic artefact caused by preceding saturated signals.

coincidence of at least 3 or 4 stations is found, a shower trigger is formed, generating a request for the buffered FADC traces from relevant stations. The response of the stations is also monitored by CDAS, which ultimately "builds" the events. The rate of the shower trigger is $\sim 0.1 \mathrm{~Hz}$. Note that, besides showers events, the CDAS records other auxiliary data-streams: the list of local triggers is continuously archived, along with control and monitoring data that describe the status of the detectors and of the CDAS.

Investigation of $S D$-rings Although $S D$-ring events are very different from those produced by air showers, we based our investigation on a similarity. $S D$-rings are characterized by the involvement of a large number of stations. This is also a characteristic of horizontal showers (zenith angle < $60^{\circ}$ ) [10], in which the number of triggered stations can reach 80 and more. In such highmultiplicity events, it may happen that two or more shower triggers are identified by CDAS, because of the transit-time of the shower over the array. This artificial splitting is corrected in the postacquisition process that merges separate triggers into a unique event, assuming that the split event 

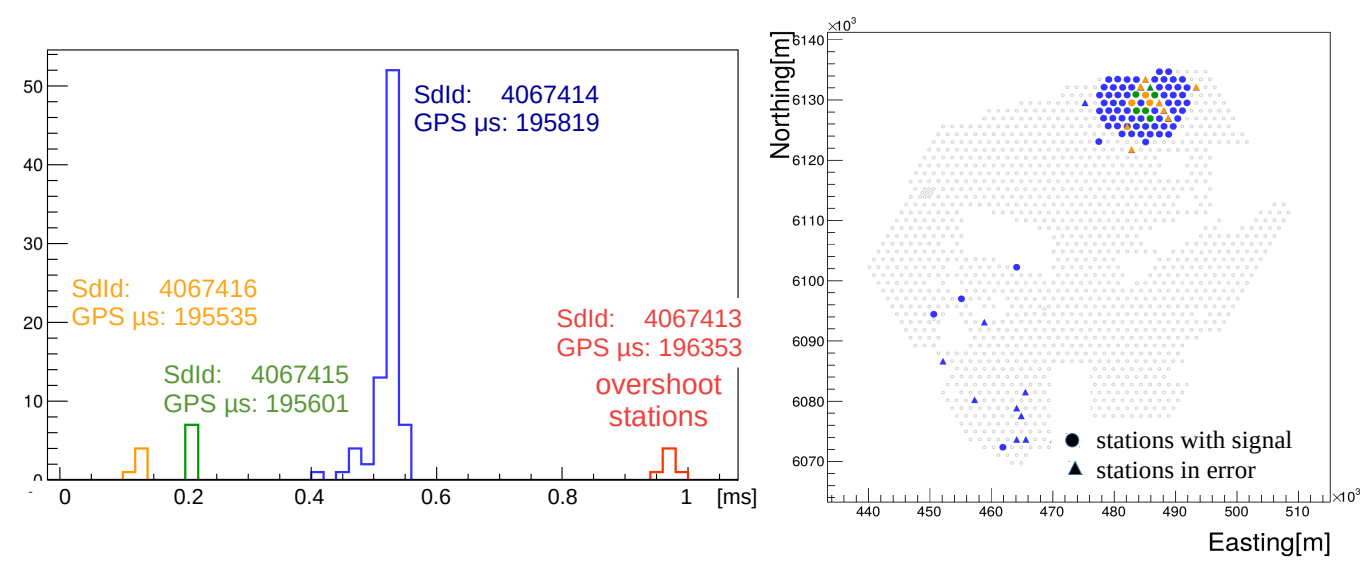

Figure 3: Left: Time sequence of the events recorded within $1 \mathrm{~ms}$ from the exemplary SD-ring (blue histogram). The late event (red histogram) includes only stations with overshoot signals. See text for details. Right: Triggered stations of the first three events. The stations of the two events preceding the SD-ring are in its footprint (blue markers). The dots represent stations that returned traces to the CDAS, while the triangles represent stations which answered with an error message.

is a shower propagating across the stations at the speed of the light. Most $S D$-rings are also the result of the merger of two or more shower triggers. Some long signals are the result of a merger too, as sometimes very long signals are divided into two trace blocks, as that shown in the panel (n) of Fig. 2, that was originally halved by the electronics as shown in (1) and (m) of the same figure.

As $S D$-rings show a longer and more complex time and space evolution than air showers, we searched for additional central triggers in a time interval of $\pm 2.5 \mathrm{~ms}$ with respect to the $S D$-ring time finding accompanying events for all the identified $S D$-rings. Inspection of these companions, together with information from the CDAS auxiliary files, allowed us to draw inferences on both the spatial and timing structure of the $S D$-rings. To illustrate our findings, and for the sake of simplicity, we discuss here only the investigation of one example, shown in Fig. 1.

Around the time of this event, we found three other triggers within $1 \mathrm{~ms}$, as shown in Fig. 3 (left), two before (orange and green histograms) and one after (red histogram). The time of the $S D$ ring is represented by the blue histogram. Each of the four histograms shows the time distribution of the stations with a signal. Remarkably the stations involved in the two preceding events are all in the footprint of the $S D$-ring, as evident in Fig. 3, right. The stations that returned successfully FADC traces (marked with dots in the figure) are due to lightning noise or muon signals. Other stations (marked with triangles) are instead in error state, meaning that they participated in the event, but they were unable to return their traces. We observed the same behaviour for all other events tagged as $S D$-rings, finding, in some cases, "long-signal" stations in the events preceding the $S D$-ring. In no case the sequence of events is "speed-of-light compatible" with a single point-source. That they happen in the very same region of the array, and in a very short time, hints at a common source generated by a phenomenon with a long time evolution. The long signals in the preceding events and in the $S D$-ring might in fact be lost for instrumental reasons.

Similarly remarkable is the event recorded about $500 \mu$ s after the $S D$-ring. This includes 13 stations, marked in the right panel of Fig. 1 with red and magenta dots: the former correspond to those that returned FADC traces to the CDAS, while the latter sent back, instead, an error message. 
As one can see, the stations in the late event are, in part, the same as in the $S D$-ring (blue markers), and, in part, they fill the central hole.

The traces from the "not-in-error" stations, however, correspond neither to showers, nor to lightning, nor to long-signals. One such signals is shown in the panel (i) of Fig. 2: it consists of a linear rise of the ADC values with little noise, the typical signal due to an overshoot of electronics. We know, from laboratory measurements, that overshoot signals may trigger the stations, and, from the electronics, we expect that the undershoot following large signals recovers and turns into an overshoot $^{1}$ after about 400 to $500 \mu$ s. In two out of the five "not-in-error" stations, we found that the late overshoot-signal corresponded to a long signal in an $S D$-ring, happened about $500 \mu$ s earlier, as expected from the response of our electronics to a large, saturated, signal. However, not all stations that were triggered by a long signal in the preceding $S D$-ring show an overshoot in the late event. A plausible explanation for this is the timing of the overshoots compared to that used to form a shower trigger. The algorithm for the latter allows for only an $8 \mu$ s time difference between neighbouring stations to form a coincidence. The probability of a station not triggering in time from an overshoot is thus fairly high, given that the time scale of the overshoot is much larger than the coincidence time that is tuned for shower signals. This also explains why no central triggers due to overshoots are found following most SD rings: if signals are not large enough and the FADCs are barely saturated, the chance of having three stations with overshoot signals in coincidence is small. In turn, not all stations with an overshoot signal in the late event, have a preceding long signal. Two of the five "not-in-error" stations were triggered in the $S D$-ring by a random muon and by a lightning. The fifth was not triggered, although we know, from analysis of the trigger-list auxiliary file, that the station was functioning at the time of detection of the SD-ring. In other words, the large signals in these three stations were missed from the data acquisition, probably due to the local trigger. This ensemble of observations, most notably that of the presence of overshoot signals following large saturated signals, allowed us to conclude that the central hole in $S D$-rings is very likely an artifact of the trigger and acquisition chain. This conclusion motivated the development of an ad-hoc local trigger discussed below.

\section{SD rings versus $S D$ disks}

While most of the 23 peculiar events are classified as $S D$-rings, a few of them, dubbed $S D$-disks, are characterised by compact footprints. Two examples are shown in Fig. 4.

Small and large $S D$-disks have similar characteristics, but differ from those of $S D$-rings. The stations involved in SD-disks exhibit long signals but all are of low amplitude. In $S D$-rings lowamplitude signals are found, in fact, but they are exceptions. Moreover, in the case of $S D$-disks, we have not found evidence of other central triggers in the same millisecond, as we did for SD-rings. To understand this difference, we have compared in detail the large $S D$-disk shown in Fig. 4, right, with an exemplary ring-event, using the CDAS archive list of all local triggers. These files store information on the station, trigger type, and trigger time. The level of trigger activity was very high in both events: ${ }^{2}$ the $S D$-ring had 591 requested traces, 278 recorded traces, 994 local triggers

\footnotetext{
${ }^{1}$ Local triggers due to overshoots, however, usually do not lead to shower triggers, because in shower events the number of stations having such a large signal is very small, mostly one.

${ }^{2}$ This amount of triggers has to be compared with the background expectation of $N_{\text {bg }} \approx 20 \mathrm{~Hz} \times 1600 \times 0.01 \mathrm{~s}=330$, and the number of T3s with the normal $\mathrm{T} 3$ rate of about $0.05 \mathrm{~Hz}$.
} 

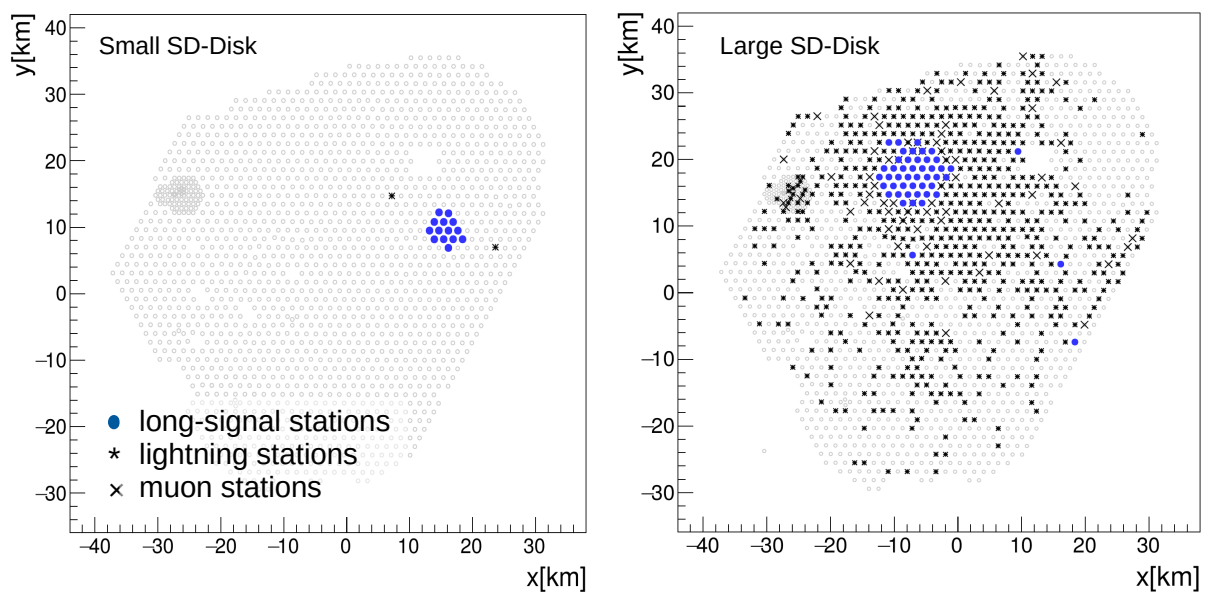

Figure 4: Footprint of a small (left) and large (right) compact event or $S D$-disk. In the latter, the long-signal stations (blue dots) are concentrated in a footprint with a radius of about $5 \mathrm{~km}$. The farthest blue dots are false positives of our selection algorithm.

in $10 \mathrm{~ms}$, while the $S D$-disk had 735 requested traces, 720 recorded traces, 1082 local triggers in $10 \mathrm{~ms}$. However, even if in the $10 \mathrm{~ms}$ interval around the trigger time of the central station there are in the $S D$-disk some 100 triggers more across the array than in the $S D$-ring, this higher number of triggers was readout in only two central triggers, compared to 11 for the $S D$-ring. This suggests a larger coherence of the signals in the $S D$-disk than in the $S D$-ring.

We have made a conservative estimate of the energy deposited in $S D$-rings and in $S D$-disks using only the signal visible in the acquired portion of trace and took the average of all stations with signal. Events with a compact footprint or small $S D$-rings (radius $<3 \mathrm{~km}$ ) are characterized by an energy deposit from 500 to $1700 \mathrm{MeV} / \mathrm{m}^{2}$, while for the $S D$-rings with a larger footprint the deposits range from 3000 to $6500 \mathrm{MeV} / \mathrm{m}^{2}$.

Finally, it is interesting to note the peculiar morphology of the $S D$-disk shown in the right panel of Fig. 4. The long-signal stations (marked by blue dots) were concentrated in a footprint with a radius of about $5 \mathrm{~km}$, but almost the whole array was in fact triggered. The signals marked with black stars were due to lightning, at the same time as the others, thus pointing to an exceptional atmospheric event.

\section{Perspectives}

While the studies presented allowed us to better understand these peculiar events, their rarity and the lack of complete information do not allow strong inferences as to their origin. Our findings hint at a link between them and TGFs. We found accompanying events within $1 \mathrm{~ms}$ of all of our $S D$-rings, and know, from previous studies [4], that the single central trigger covers tens of microseconds. These observations are compatible with the evolution of the lightning leaders associated with TGFs. Moreover the presence of low clouds at the time of some of events is consistent with the expectations for downward TGFs. From laser and LIDAR measurements, we know that at the time of some other events, such as that in Fig. 4-right, the clouds were higher. One could infer that the footprint is larger at ground as the altitude of the source increases. If we consider additionally the lightning stations, the high rate of which, during thunderstorms, may have prevented the acquisition of long 
signals in the external part of the event, the footprint of this event could be much larger. Finally, the observed peculiar events seem to be intense phenomena, with energy deposits two orders of magnitude larger than in a vertical shower initiated by a particle of $10^{19} \mathrm{eV}$. We find the energy density to be comparable to that measured at $650 \mathrm{~m}$ from the source with NaI scintillators by Dwyer et al. [12]. NaI has an efficient response to photons as do the water-Cherenkov detectors.

The collection of a larger number of such events, and especially of more complete ones, is key to interpret these events. The 23 events detected at the observatory are actually not uniformly distributed in time because of a change to the shower trigger system in 2014 to enhance detection of low energy events. This raised the number of central triggers during periods with lightning activity perhaps explaining the observed decrease in the rate of $S D$-rings. This problem could be reduced by modifying the CDAS read-out logic to give priority to events which contain long signals. An algorithm to tag such kind of signals has been recently developed using differences of integrals performed over predefined parts of the trace. The concept is that the oscillating features of the lightning noise will average out if the integral is used instead of the peak. Moreover, the trigger algorithm will be independent of the baseline, making it robust against undershoots or overshoots. This change has been implemented in a small part of the SD array. No problems in the normal data-taking were observed. We plan to extend it to the whole array before the next austral summer, when intense thunderstorms are expected.

Crucial to the understanding of the phenomenon creating the peculiar events will be the exploitation of data from instruments recently installed at the observatory that can monitor lightning and electric field at ground, such as Boltek Storm trackers and E-field mills. Measurements can be correlated with the information from the water-Cherenkov detectors and from the scintillators being installed above each SD station. Finally, the radio antennas that form the Auger Engineering Radio Array, as well as those that will be installed on each SD stations, can also be used as sensors of atmospheric electricity.

Acknowledgments The authors wish to thank the World Wide Lightning Location Network (wwlln.net), for providing the lightning location data used in this paper.

\section{References}

[1] Pierre Auger Coll., Nucl. Instrum. Meth. A 798 (2015) 172-213, [1502. 01323].

[2] Pierre Auger Coll., Earth Space Sci. 7 (2020) e2019EA000582.

[3] A. Vásquez-Ramírez [for the Pierre Auger Coll.], these proceedings.

[4] R. Colalillo [for the Pierre Auger Coll.], Proc. 35th Int. Cosmic Ray Conf., Busan, Korea (2017), PoS(ICRC2017)314, [1708.06592].

[5] R. Colalillo [for the Pierre Auger Coll.], EPJ Web Conf. 197 (2019) 03003, AtmoHEAD 2018.

[6] J.W. Belz et al. [Telescope Array Coll.], J. Geophys. Res.-Atmos. 125 (2020) e2019JD031940.

[7] J.R. Dwyer, D.M. Smith, and S.A. Cummer, Space Sci. Rev. 173 (2012) 133-196.

[8] Y. Wada et al., Phys. Commun. 2 (2019) 67.

[9] Pierre Auger Coll., Nucl. Instrum. Meth. A 613 (2010) 29.

[10] Pierre Auger Coll., J. Cosmol. Astropart. Phys. 08 (2014) 019.

[11] B. Keilhauer [for the Pierre Auger Coll.], EPJ Web Conf. 197 (2019) 02001, AtmoHEAD 2018.

[12] J.R. Dwyer et al., Geophys. Res. Lett. 31 (2004) L05119. 


\section{The Pierre Auger Collaboration}

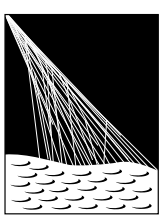

PIERRE

AUSGERVATORY

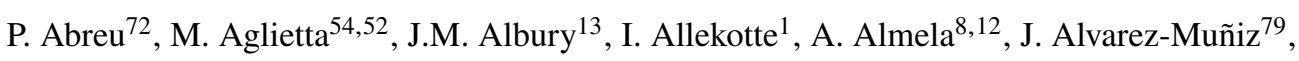
R. Alves Batista ${ }^{80}$, G.A. Anastasi ${ }^{63,52}$, L. Anchordoqui ${ }^{87}$, B. Andrada ${ }^{8}$, S. Andringa ${ }^{72}$, C. $\mathrm{Aramo}^{50}$, P.R. Araújo Ferreira ${ }^{42}$, J. C. Arteaga Velázquez ${ }^{67}$, H. Asorey $^{8}$, P. Assis ${ }^{72}$, G. Avila ${ }^{11}$, A.M. Badescu ${ }^{75}$, A. Bakalova ${ }^{32}$, A. Balaceanu ${ }^{73}$, F. Barbato ${ }^{45,46}$, R.J. Barreira Luz $^{72}$, K.H. Becker ${ }^{38}$, J.A. Bellido ${ }^{13,69}$, C. Berat ${ }^{36}$, M.E. Bertaina ${ }^{63,52}$, X. Bertou ${ }^{1}$, P.L. Biermann ${ }^{b}$, V. Binet ${ }^{6}$, K. Bismark ${ }^{39,8}$, T. Bister ${ }^{42}$, J. Biteau ${ }^{37}$, J. Blazek ${ }^{32}$, C. Bleve ${ }^{36}$, M. Boháčová ${ }^{32}$, D. Boncioli ${ }^{57,46}$, C. Bonifazi ${ }^{9,26}$, L. Bonneau Arbeletche ${ }^{21}$, N. Borodai ${ }^{70}$, A.M. Botti ${ }^{8}$, J. Brack ${ }^{d}$, T. Bretz ${ }^{42}$, P.G. Brichetto Orchera ${ }^{8}$, F.L. Briechle ${ }^{42}$, P. Buchholz ${ }^{44}$, A. Bueno ${ }^{78}$, S. Buitink ${ }^{15}$, M. Buscemi ${ }^{47}$, M. Büsken ${ }^{39,8}$, K.S. Caballero-Mora ${ }^{66}$, L. Caccianiga ${ }^{59,49}$, F. Canfora ${ }^{80,81}$, I. Caracas ${ }^{38}$, J.M. Carceller ${ }^{78}$, R. Caruso ${ }^{58,47}$, A. Castellina ${ }^{54,52}$, F. Catalanii ${ }^{19}$, G. Cataldi ${ }^{48}$, L. Cazon ${ }^{72}$, M. Cerda ${ }^{10}$, J.A. Chinellato ${ }^{22}$, J. Chudoba ${ }^{32}$, L. Chytka ${ }^{33}$, R.W. Clay ${ }^{13}$, A.C. Cobos Ceruttii ${ }^{7}$, R. Colalillo ${ }^{60,50}$, A. Coleman ${ }^{93}$, M.R. Coluccia ${ }^{48}$, R. Conceição ${ }^{72}$, A. Condorelli ${ }^{45,46}$, G. Consolati ${ }^{49,55}$, F. Contreras ${ }^{11}$, F. Convenga ${ }^{56,48}$, D. Correia dos Santos $^{28}$, C.E. Covault ${ }^{85}$, S. Dasso ${ }^{5,3}$, K. Daumiller ${ }^{41}$, B.R. Dawson ${ }^{13}$, J.A. Day ${ }^{13}$, R.M. de Almeida $^{28}$, J. de Jesús ${ }^{8,41}$, S.J. de Jong ${ }^{80,81}$, G. De Mauro ${ }^{80,81}$, J.R.T. de Mello Neto ${ }^{26,27}$, I. De Mitri ${ }^{45,46}$, J. de Oliveira ${ }^{18}$, D. de Oliveira Franco ${ }^{22}$, F. de Palma ${ }^{56,48}$, V. de Souza $^{20}$, E. De Vito ${ }^{56,48}$, M. del Río ${ }^{11}$, O. Deligny ${ }^{34}$, L. Deval ${ }^{41,8}$, A. di Matteo $^{52}$, C. Dobrigkeit ${ }^{22}$, J.C. D’Olivo ${ }^{68}$, L.M. Domingues Mendes ${ }^{72}$, R.C. dos Anjos ${ }^{25}$, D. dos Santos $^{28}$, M.T. Dova ${ }^{4}$, J. Ebr ${ }^{32}$, R. Engel ${ }^{39,41}$, I. Epicoco ${ }^{56,48}$, M. Erdmann ${ }^{42}$, C.O. Escobar ${ }^{a}$, A. Etchegoyen ${ }^{8,12}$, H. Falcke ${ }^{80,82,81}$, J. Farmer ${ }^{92}$, G. Farrar ${ }^{90}$, A.C. Fauth ${ }^{22}$, N. Fazzini ${ }^{a}$, F. Feldbusch ${ }^{40}$, F. Fenu ${ }^{54,52}$,

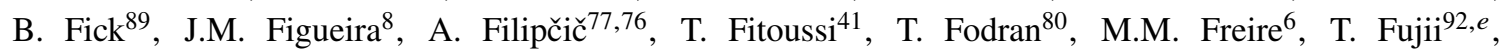
A. Fuster $^{8,12}$, C. Galea ${ }^{80}$, C. Galelli ${ }^{59,49}$, B. García ${ }^{7}$, A.L. Garcia Vegas ${ }^{42}$, H. Gemmeke ${ }^{40}$, F. Gesualdi ${ }^{8,41}$, A. Gherghel-Lascu ${ }^{73}$, P.L. Ghia ${ }^{34}$, U. Giaccari ${ }^{80}$, M. Giammarchi ${ }^{49}$, J. Glombitza ${ }^{42}$, F. Gobbi ${ }^{10}$, F. Gollan ${ }^{8}$, G. Golup ${ }^{1}$, M. Gómez Berisso ${ }^{1}$, P.F. Gómez Vitale ${ }^{11}$, J.P. Gongora ${ }^{11}$, J.M. González ${ }^{1}$, N. González ${ }^{14}$, I. Goos ${ }^{1,41}$, D. Góra ${ }^{70}$, A. Gorgi ${ }^{54,52}$, M. Gottowik ${ }^{38}$, T.D. Grubb ${ }^{13}$, F. Guarino ${ }^{60,50}$, G.P. Guedes ${ }^{23}$, E. Guido ${ }^{52,63}$, S. $\mathrm{Hahn}^{41,8}$, P. $\mathrm{Hamal}^{32}$, M.R. Hampel ${ }^{8}$, P. Hansen ${ }^{4}$, D. Harari ${ }^{1}$, V.M. Harvey ${ }^{13}$, A. Haungs ${ }^{41}$, T. Hebbeker ${ }^{42}$, D. Heck ${ }^{41}$, G.C. Hill ${ }^{13}$, C. Hojvat ${ }^{a}$, J.R. Hörandel ${ }^{80,81}$, P. Horvath ${ }^{33}$, M. Hrabovský ${ }^{33}$, T. Huege ${ }^{41,15}$, A. Insolia ${ }^{58,47}$, P.G. Isar $^{74}$, P. Janecek ${ }^{32}$, J.A. Johnsen ${ }^{86}$, J. Jurysek ${ }^{32}$, A. Kääpä ${ }^{38}$, K.H. Kampert ${ }^{38}$, N. Karastathis ${ }^{41}$, B. Keilhauer ${ }^{41}$, J. Kemp ${ }^{42}$, A. Khakurdikar ${ }^{80}$, V.V. Kizakke Covilakam $^{8,41}$, H.O. Klages ${ }^{41}$, M. Kleifges ${ }^{40}$, J. Kleinfeller ${ }^{10}$, M. Köpke ${ }^{39}$, N. Kunka ${ }^{40}$, B.L. Lago ${ }^{17}$, R.G. Lang ${ }^{20}$, N. Langner ${ }^{42}$, M.A. Leigui de Oliveira ${ }^{24}$, V. Lenok $^{41}$, A. Letessier-Selvon ${ }^{35}$, I. LhenryYvon $^{34}$, D. Lo Presti ${ }^{58,47}$, L. Lopes ${ }^{72}$, R. López ${ }^{64}$, L. Lu ${ }^{94}$, Q. Luce ${ }^{39}$, J.P. Lundquist ${ }^{76}$, A. Machado Payeras $^{22}$, G. Mancarella ${ }^{56,48}$, D. Mandat ${ }^{32}$, B.C. Manning ${ }^{13}$, J. Manshanden ${ }^{43}$, P. Mantsch ${ }^{a}$, S. Marafico ${ }^{34}$, A.G. Mariazzi ${ }^{4}$, I.C. Mariş ${ }^{14}$, G. Marsella ${ }^{61,47}$, D. Martello ${ }^{56,48}$, S. Martinelli ${ }^{41,8}$, O. Martínez Bravo ${ }^{64}$, M. Mastrodicasa ${ }^{57,46}$, H.J. Mathes ${ }^{41}$, J. Matthews ${ }^{88}$, G. Matthiae ${ }^{62,51}$, E. Mayotte ${ }^{38}$, P.O. Mazur ${ }^{a}$, G. MedinaTanco $^{68}$, D. Melo $^{8}$, A. Menshikov ${ }^{40}$, K.-D. Merenda ${ }^{86}$, S. Michal ${ }^{33}$, M.I. Micheletti ${ }^{6}$, L. Miramonti ${ }^{59,49}$, S. Mollerach ${ }^{1}$, F. Montanet ${ }^{36}$, C. Morello ${ }^{54,52}$, M. Mostafá ${ }^{91}$, A.L. Müller ${ }^{8}$, M.A. Muller ${ }^{22}$, K. Mulrey ${ }^{15}$, R. Mussa ${ }^{52}$, M. Muzio ${ }^{90}$, W.M. Namasaka ${ }^{38}$, A. Nasr-Esfahani ${ }^{38}$, L. Nellen ${ }^{68}$, M. Niculescu-Oglinzanu ${ }^{73}$, M. Niechciol ${ }^{44}$, D. Nitz ${ }^{89}$, D. Nosek ${ }^{31}$, V. Novotny ${ }^{31}$, L. Nožka ${ }^{33}$, A Nucita ${ }^{56,48}$, L.A. Núñez ${ }^{30}$, M. Palatka ${ }^{32}$, J. Pallotta ${ }^{2}$, P. Papenbreer ${ }^{38}$, G. Parente ${ }^{79}$, A. Parra ${ }^{64}$, J. Pawlowsky ${ }^{38}$, M. Pech ${ }^{32}$, F. Pedreira ${ }^{79}$, J. Pȩkala ${ }^{70}$, R. Pelayo ${ }^{65}$, J. Peña-Rodriguez ${ }^{30}$, E.E. Pereira Martins ${ }^{39,8}$, J. Perez Armand ${ }^{21}$, C. Pérez Bertolli $^{8,41}$, M. Perlin ${ }^{8,41}$, L. Perrone ${ }^{56,48}$, S. Petrera ${ }^{45,46}$, T. Pierog ${ }^{41}$, M. Pimenta ${ }^{72}$, V. Pirronello ${ }^{58,47}$, M. Platino ${ }^{8}$, B. Pont $^{80}$, M. Pothast ${ }^{81,80}$, P. Privitera ${ }^{92}$, M. Prouza ${ }^{32}$, A. Puyleart ${ }^{89}$, S. Querchfeld ${ }^{38}$, J. Rautenberg ${ }^{38}$, D. Ravignani ${ }^{8}$, M. Reininghaus ${ }^{41,8}$, J. Ridky ${ }^{32}$, F. Riehn ${ }^{72}$, M. Risse ${ }^{44}$, V. Rizi ${ }^{57,46}$, W. Rodrigues de Carvalho ${ }^{21}$, J. Rodriguez Rojo ${ }^{11}$, M.J. Roncoroni ${ }^{8}$, S. Rossoni ${ }^{43}$, M. Roth ${ }^{41}$, E. Roulet ${ }^{1}$, A.C. Rovero ${ }^{5}$, P. Ruehl ${ }^{44}$, A. Saftoiu ${ }^{73}$, F. Salamida ${ }^{57,46}$, H. Salazar ${ }^{64}$, G. Salina ${ }^{51}$, J.D. Sanabria Gomez ${ }^{30}$, F. Sánchez ${ }^{8}$, E.M. Santos ${ }^{21}$, E. Santos ${ }^{32}$, F. Sarazin ${ }^{86}$, R. Sarmento ${ }^{72}$, C. Sarmiento-Cano ${ }^{8}$, R. Sato ${ }^{11}$, 
P. Savina ${ }^{56,48,34,94}$, C.M. Schäfer ${ }^{41}$, V. Scherini ${ }^{56,48}$, H. Schieler ${ }^{41}$, M. Schimassek ${ }^{39,8}$, M. Schimp ${ }^{38}$, F. Schlüter ${ }^{41,8}$, D. Schmidt ${ }^{39}$, O. Scholten ${ }^{84,15}$, P. Schovánek ${ }^{32}$, F.G. Schröder ${ }^{93,41}$, S. Schröder ${ }^{38}$, J. Schulte ${ }^{42}$, S.J. Sciutto ${ }^{4}$, M. Scornavacche ${ }^{8,41}$, A. Segreto ${ }^{53,47}$, S. Sehgal ${ }^{38}$, R.C. Shellard ${ }^{16}$, G. Sigl ${ }^{43}$, G. Silli ${ }^{8,41}$, O. Sima ${ }^{73, f}$, R. Šmída ${ }^{92}$, P. Sommers ${ }^{91}$, J.F. Soriano ${ }^{87}$, J. Souchard ${ }^{36}$, R. Squartini ${ }^{10}$, M. Stadelmaier ${ }^{41,8}$, D. Stanca ${ }^{73}$, S. Stanič ${ }^{76}$, J. Stasielak ${ }^{70}$, P. Stassi ${ }^{36}$, A. Streich ${ }^{39,8}$, M. Suárez-Durán ${ }^{14}$, T. Sudholz ${ }^{13}$, T. Suomijärvi ${ }^{37}$, A.D. Supanitsky ${ }^{8}$, Z. Szadkowski ${ }^{71}$, A. Tapia ${ }^{29}$, C. Taricco ${ }^{63,52}$, C. Timmermans ${ }^{81,80}$, O. Tkachenko ${ }^{41}$, P. Tobiska ${ }^{32}$, C.J. Todero Peixoto ${ }^{19}$, B. Tomé ${ }^{72}$, Z. Torrès ${ }^{36}$, A. Travaini ${ }^{10}$, P. Travnicek $^{32}$, C. Trimarelli ${ }^{57,46}$, M. Tueros ${ }^{4}$, R. Ulrich ${ }^{41}$, M. Unger ${ }^{41}$, L. Vaclavek ${ }^{33}$, M. Vacula ${ }^{33}$, J.F. Valdés Galicia ${ }^{68}$, L. Valore ${ }^{60,50}$, E. Varela ${ }^{64}$, A. Vásquez-Ramírez ${ }^{30}$, D. Veberič ${ }^{41}$, C. Ventura ${ }^{27}$, I.D. Vergara Quispe ${ }^{4}$, V. Verzi ${ }^{51}$, J. Vicha ${ }^{32}$, J. Vink $^{83}$, S. Vorobiov ${ }^{76}$, H. Wahlberg ${ }^{4}$, C. Watanabe ${ }^{26}$, A.A. Watson ${ }^{c}$, M. Weber ${ }^{40}$, A. Weindl ${ }^{41}$, L. Wiencke ${ }^{86}$, H. Wilczyński ${ }^{70}$, M. Wirtz ${ }^{42}$, D. Wittkowski ${ }^{38}$, B. Wundheiler ${ }^{8}$, A. Yushkov $^{32}$, O. Zapparrata ${ }^{14}$, E. Zas ${ }^{79}$, D. Zavrtanik ${ }^{76,77}$, M. Zavrtanik ${ }^{77,76}$, L. Zehrer ${ }^{76}$

${ }^{1}$ Centro Atómico Bariloche and Instituto Balseiro (CNEA-UNCuyo-CONICET), San Carlos de Bariloche, Argentina

${ }^{2}$ Centro de Investigaciones en Láseres y Aplicaciones, CITEDEF and CONICET, Villa Martelli, Argentina

${ }^{3}$ Departamento de Física and Departamento de Ciencias de la Atmósfera y los Océanos, FCEyN, Universidad de Buenos Aires and CONICET, Buenos Aires, Argentina

${ }^{4}$ IFLP, Universidad Nacional de La Plata and CONICET, La Plata, Argentina

${ }^{5}$ Instituto de Astronomía y Física del Espacio (IAFE, CONICET-UBA), Buenos Aires, Argentina

${ }^{6}$ Instituto de Física de Rosario (IFIR) - CONICET/U.N.R. and Facultad de Ciencias Bioquímicas y Farmacéuticas U.N.R., Rosario, Argentina

${ }^{7}$ Instituto de Tecnologías en Detección y Astropartículas (CNEA, CONICET, UNSAM), and Universidad Tecnológica Nacional - Facultad Regional Mendoza (CONICET/CNEA), Mendoza, Argentina

${ }^{8}$ Instituto de Tecnologías en Detección y Astropartículas (CNEA, CONICET, UNSAM), Buenos Aires, Argentina

${ }^{9}$ International Center of Advanced Studies and Instituto de Ciencias Físicas, ECyT-UNSAM and CONICET, Campus Miguelete - San Martín, Buenos Aires, Argentina

${ }^{10}$ Observatorio Pierre Auger, Malargüe, Argentina

${ }^{11}$ Observatorio Pierre Auger and Comisión Nacional de Energía Atómica, Malargüe, Argentina

12 Universidad Tecnológica Nacional - Facultad Regional Buenos Aires, Buenos Aires, Argentina

${ }^{13}$ University of Adelaide, Adelaide, S.A., Australia

14 Université Libre de Bruxelles (ULB), Brussels, Belgium

15 Vrije Universiteit Brussels, Brussels, Belgium

${ }^{16}$ Centro Brasileiro de Pesquisas Fisicas, Rio de Janeiro, RJ, Brazil

${ }^{17}$ Centro Federal de Educação Tecnológica Celso Suckow da Fonseca, Nova Friburgo, Brazil

${ }^{18}$ Instituto Federal de Educação, Ciência e Tecnologia do Rio de Janeiro (IFRJ), Brazil

${ }^{19}$ Universidade de São Paulo, Escola de Engenharia de Lorena, Lorena, SP, Brazil

${ }^{20}$ Universidade de São Paulo, Instituto de Física de São Carlos, São Carlos, SP, Brazil

${ }^{21}$ Universidade de São Paulo, Instituto de Física, São Paulo, SP, Brazil

${ }^{22}$ Universidade Estadual de Campinas, IFGW, Campinas, SP, Brazil

${ }^{23}$ Universidade Estadual de Feira de Santana, Feira de Santana, Brazil

${ }^{24}$ Universidade Federal do ABC, Santo André, SP, Brazil

${ }^{25}$ Universidade Federal do Paraná, Setor Palotina, Palotina, Brazil

${ }^{26}$ Universidade Federal do Rio de Janeiro, Instituto de Física, Rio de Janeiro, RJ, Brazil

${ }^{27}$ Universidade Federal do Rio de Janeiro (UFRJ), Observatório do Valongo, Rio de Janeiro, RJ, Brazil

${ }^{28}$ Universidade Federal Fluminense, EEIMVR, Volta Redonda, RJ, Brazil

${ }^{29}$ Universidad de Medellín, Medellín, Colombia

${ }^{30}$ Universidad Industrial de Santander, Bucaramanga, Colombia

${ }^{31}$ Charles University, Faculty of Mathematics and Physics, Institute of Particle and Nuclear Physics, Prague, Czech Republic

32 Institute of Physics of the Czech Academy of Sciences, Prague, Czech Republic 
${ }^{33}$ Palacky University, RCPTM, Olomouc, Czech Republic

34 CNRS/IN2P3, IJCLab, Université Paris-Saclay, Orsay, France

${ }^{35}$ Laboratoire de Physique Nucléaire et de Hautes Energies (LPNHE), Sorbonne Université, Université de Paris, CNRSIN2P3, Paris, France

${ }^{36}$ Univ. Grenoble Alpes, CNRS, Grenoble Institute of Engineering Univ. Grenoble Alpes, LPSC-IN2P3, 38000 Grenoble, France

${ }^{37}$ Université Paris-Saclay, CNRS/IN2P3, IJCLab, Orsay, France

38 Bergische Universität Wuppertal, Department of Physics, Wuppertal, Germany

${ }^{39}$ Karlsruhe Institute of Technology (KIT), Institute for Experimental Particle Physics, Karlsruhe, Germany

${ }^{40}$ Karlsruhe Institute of Technology (KIT), Institut für Prozessdatenverarbeitung und Elektronik, Karlsruhe, Germany

${ }^{41}$ Karlsruhe Institute of Technology (KIT), Institute for Astroparticle Physics, Karlsruhe, Germany

${ }^{42}$ RWTH Aachen University, III. Physikalisches Institut A, Aachen, Germany

43 Universität Hamburg, II. Institut für Theoretische Physik, Hamburg, Germany

${ }^{44}$ Universität Siegen, Department Physik - Experimentelle Teilchenphysik, Siegen, Germany

${ }^{45}$ Gran Sasso Science Institute, L'Aquila, Italy

46 INFN Laboratori Nazionali del Gran Sasso, Assergi (L'Aquila), Italy

${ }^{47}$ INFN, Sezione di Catania, Catania, Italy

${ }^{48}$ INFN, Sezione di Lecce, Lecce, Italy

${ }^{49}$ INFN, Sezione di Milano, Milano, Italy

${ }^{50}$ INFN, Sezione di Napoli, Napoli, Italy

${ }^{51}$ INFN, Sezione di Roma "Tor Vergata", Roma, Italy

52 INFN, Sezione di Torino, Torino, Italy

53 Istituto di Astrofisica Spaziale e Fisica Cosmica di Palermo (INAF), Palermo, Italy

54 Osservatorio Astrofisico di Torino (INAF), Torino, Italy

55 Politecnico di Milano, Dipartimento di Scienze e Tecnologie Aerospaziali, Milano, Italy

56 Università del Salento, Dipartimento di Matematica e Fisica "E. De Giorgi”, Lecce, Italy

57 Università dell'Aquila, Dipartimento di Scienze Fisiche e Chimiche, L'Aquila, Italy

58 Università di Catania, Dipartimento di Fisica e Astronomia, Catania, Italy

${ }^{59}$ Università di Milano, Dipartimento di Fisica, Milano, Italy

${ }^{60}$ Università di Napoli "Federico II", Dipartimento di Fisica "Ettore Pancini”, Napoli, Italy

${ }^{61}$ Università di Palermo, Dipartimento di Fisica e Chimica "E. Segrè", Palermo, Italy

62 Università di Roma "Tor Vergata", Dipartimento di Fisica, Roma, Italy

${ }^{63}$ Università Torino, Dipartimento di Fisica, Torino, Italy

${ }^{64}$ Benemérita Universidad Autónoma de Puebla, Puebla, México

${ }^{65}$ Unidad Profesional Interdisciplinaria en Ingeniería y Tecnologías Avanzadas del Instituto Politécnico Nacional (UPIITA-IPN), México, D.F., México

66 Universidad Autónoma de Chiapas, Tuxtla Gutiérrez, Chiapas, México

${ }^{67}$ Universidad Michoacana de San Nicolás de Hidalgo, Morelia, Michoacán, México

${ }^{68}$ Universidad Nacional Autónoma de México, México, D.F., México

${ }^{69}$ Universidad Nacional de San Agustin de Arequipa, Facultad de Ciencias Naturales y Formales, Arequipa, Peru

${ }^{70}$ Institute of Nuclear Physics PAN, Krakow, Poland

${ }^{71}$ University of Łódź, Faculty of High-Energy Astrophysics, Łódź, Poland

${ }^{72}$ Laboratório de Instrumentação e Física Experimental de Partículas - LIP and Instituto Superior Técnico - IST, Universidade de Lisboa - UL, Lisboa, Portugal

73 "Horia Hulubei” National Institute for Physics and Nuclear Engineering, Bucharest-Magurele, Romania

${ }^{74}$ Institute of Space Science, Bucharest-Magurele, Romania

75 University Politehnica of Bucharest, Bucharest, Romania

76 Center for Astrophysics and Cosmology (CAC), University of Nova Gorica, Nova Gorica, Slovenia

${ }^{77}$ Experimental Particle Physics Department, J. Stefan Institute, Ljubljana, Slovenia

78 Universidad de Granada and C.A.F.P.E., Granada, Spain

${ }^{79}$ Instituto Galego de Física de Altas Enerxías (IGFAE), Universidade de Santiago de Compostela, Santiago de Compostela, Spain 
${ }^{80}$ IMAPP, Radboud University Nijmegen, Nijmegen, The Netherlands

${ }^{81}$ Nationaal Instituut voor Kernfysica en Hoge Energie Fysica (NIKHEF), Science Park, Amsterdam, The Netherlands

82 Stichting Astronomisch Onderzoek in Nederland (ASTRON), Dwingeloo, The Netherlands

${ }^{83}$ Universiteit van Amsterdam, Faculty of Science, Amsterdam, The Netherlands

${ }^{84}$ University of Groningen, Kapteyn Astronomical Institute, Groningen, The Netherlands

85 Case Western Reserve University, Cleveland, OH, USA

86 Colorado School of Mines, Golden, CO, USA

${ }^{87}$ Department of Physics and Astronomy, Lehman College, City University of New York, Bronx, NY, USA

${ }^{88}$ Louisiana State University, Baton Rouge, LA, USA

${ }^{89}$ Michigan Technological University, Houghton, MI, USA

${ }^{90}$ New York University, New York, NY, USA

${ }^{91}$ Pennsylvania State University, University Park, PA, USA

92 University of Chicago, Enrico Fermi Institute, Chicago, IL, USA

93 University of Delaware, Department of Physics and Astronomy, Bartol Research Institute, Newark, DE, USA

94 University of Wisconsin-Madison, Department of Physics and WIPAC, Madison, WI, USA

${ }^{a}$ Fermi National Accelerator Laboratory, Fermilab, Batavia, IL, USA

${ }^{b}$ Max-Planck-Institut für Radioastronomie, Bonn, Germany

${ }^{c}$ School of Physics and Astronomy, University of Leeds, Leeds, United Kingdom

${ }^{d}$ Colorado State University, Fort Collins, CO, USA

$e^{e}$ now at Hakubi Center for Advanced Research and Graduate School of Science, Kyoto University, Kyoto, Japan

$f$ also at University of Bucharest, Physics Department, Bucharest, Romania 